\title{
Investigation of synthetic spider silk crystallinity and alignment via electrothermal, pyroelectric, literature XRD, and tensile techniques
}

Troy Munro

Mechanical Engineering Department, Brigham Young University, troy.munro@byu.edu

Tristan Putzeys

Laboratory for Soft matter and Biophysics, Department of Physics and Astronomy, KU Leuven

Michael Wubbenhorst

Laboratory for Soft matter and Biophysics, Department of Physics and Astronomy, KU Leuven

Eq/lpst thisriend additional works at: https://scholarsarchive.byu.edu/facpub

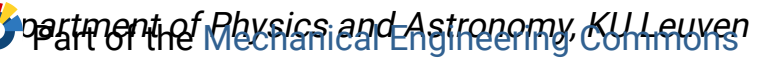

Cameron G. Copeland

Qriggiatat BibplicationeGitationgy Dept., Utah State University

Munro, T., Putzeys, T., Copeland, C., Xing, C., Lewis, R., Ban, H., Glorieux, C., and Wubbenhorst, M., "Investigation of synthetic spider silk crystallinity and alignment via electrothermal, pyroelectric, See next page for additional authors

literature XRD, and tensile techniques," Macromolecular Materials and Engineering, DOI:

10.1002/mame.201600480, 2017.

\section{BYU ScholarsArchive Citation}

Munro, Troy; Putzeys, Tristan; Wubbenhorst, Michael; Glorieux, Christ; Copeland, Cameron G.; Lewis, Randolph V.; Xing, Changhu; and Ban, Heng, "Investigation of synthetic spider silk crystallinity and alignment via electrothermal, pyroelectric, literature XRD, and tensile techniques" (2017). Faculty Publications. 1876.

https://scholarsarchive. byu.edu/facpub/1876

This Peer-Reviewed Article is brought to you for free and open access by BYU ScholarsArchive. It has been accepted for inclusion in Faculty Publications by an authorized administrator of BYU ScholarsArchive. For more information, please contact ellen_amatangelo@byu.edu. 


\section{Authors}

Troy Munro, Tristan Putzeys, Michael Wubbenhorst, Christ Glorieux, Cameron G. Copeland, Randolph V. Lewis, Changhu Xing, and Heng Ban 


\section{WILEY-VCH}

DOI: 10.1002/ ((mame.201600480))

Article type: ((Full Paper))

\section{Investigation of synthetic spider silk crystallinity and alignment via electrothermal, pyroelectric, literature XRD, and tensile techniques}

Troy Munro, Tristan Putzeys, Cameron G. Copeland, Changhu Xing, Randolph V Lewis, Heng Ban, Christ Glorieux, Michael Wubbenhorst

Dr. T. Munro

Mechanical Engineering Department, Brigham Young University, Provo, UT 84602, USA E-mail: troy.munro@byu.edu

Dr. T. Putzeys, Dr. C. Glorieux, Dr. M. Wubbenhorst

Laboratory for Soft Matter and Biophysics, Department of Physics and Astronomy, KU Leuven, Heverlee B-3001, Belgium

Dr. T. Putzeys, Functional Organic Materials and Devices, Department of Chemical Engineering and Chemistry, TU/e Eindhoven University of Technology, 5600 MB Eindhoven, the Netherlands

Dr. C.G. Copeland, Dr. R.V. Lewis

Synthetic Bioproducts Center, Biology Dept., Utah State University, North Logan, UT 84341, USA

Dr. C. Xing, Dr. H. Ban

Mechanical and Aerospace Engineering Department, Utah State University, Logan, UT 84322, USA

Keywords: spider silk, crystallinity, thermal, pyroelectric, processing

The processes used to create synthetic spider silk greatly affect the properties of the produced fibers. This paper investigates the effect of process variations during artificial spinning on the thermal and mechanical properties of the produced silk. Property values are also compared to the ones of the natural dragline silk of the $N$. clavipes spider, and to unprocessed (as-spun) synthetic silk. Structural characterization by scanning pyroelectric microscopy is employed to provide insight into the axial orientation of the crystalline regions of the fiber and is supported by XRD data. The results show that stretching and passage through liquid baths induce crystal formation and axial alignment in synthetic fibers, but with different structural organization than natural silks. Furthermore, an increase in thermal diffusivity and elastic modulus is observed with decreasing fiber diameter, trending towards properties of natural fiber. This effect seems to be related to silk fibers being subjected to a radial gradient during production.

\section{Introduction}

In the past decade, a significant research interest has emerged toward spiders and spider silk because of their exceptional mechanical properties ${ }^{[1,2]}$. A single orb weaving spider is capable of spinning a multitude of silk types, each emerging from its own particular set of abdominal 


\section{WILEY-VCH}

silk glands, which enable the spider to tailor silk to a single specific task (e.g. web assembly, egg-case construction, prey wrapping, transportation, etc. ${ }^{[2-4]}$. Most silks, in particular dragline silk from the major ampullate gland, possess revolutionary mechanical properties due to their unique combination of high tensile strength with large ductility ${ }^{[5]}$.

However, the low yield of harvesting silk from either free or caged spiders limits natural spider silk as a construction material for economic reasons. To overcome this obstacle, numerous processes exist to produce synthetic spider silk, with properties close to the ones of natural spider silk.

The process to artificially synthesize spider silk into fibers consists of two consecutive pathways: the synthesis of the feedstock (the unspun silk dope in spiders) and mimicking the mechanical spinning conditions (the funnel, valve, tapering duct, and spigot ${ }^{[6]}$. For protein production, the synthetic protein host can limit the size of the repeated amino acid sequence, limiting the size of the expressed protein ${ }^{[7]}$, which can affect the fiber spinning and the fiber's properties. In 2000, the Canadian biotechnology company Nexia successfully produced spider silk protein in transgenic goats that carried the gene for it, where the milk produced by the goats contained significant quantities of the two proteins present in the natural silk ${ }^{[8,9]}$. The Lewis Spider Silk Lab has produced fibers from recombinant spider silk protein from transgenic goats ${ }^{[10-13]}$.

Mimicking the natural spinning process of the spider is an ongoing challenge for spider silk production. The protein dope (composed of both MaSp1 and MaSp2 proteins ${ }^{[14,15]}$ ) inside the spider is water-soluble, but until recently ${ }^{[}$, recombinant proteins have only been solubilized in a chaotropic agent, HFIP (hexafluoroisopropanol), at concentrations lower than the ones found naturally in the spider. Recent work has successfully spun recombinant proteins from aqueous solutions rather than $\operatorname{HFIP}^{[9,16-18]}$ where significant fluctuations in the mechanical properties were observed due to the non-optimized process for fiber and film production. The spinning of the protein dope into a usable fiber must then attempt to mimic the processes in the spider ducts where water is removed and the proteins folds into a water-insoluble fiber ${ }^{[6]}$. Microfluidic channels (where ionic concentrations and $\mathrm{pH}$ can be carefully controlled) have been able to produce small quantities of synthetic silk ${ }^{[18,19]}$, but this process is difficult to scale-up for the commercial production of spider silk. A continuous extrusion machine has been used to successfully produce large quantities ( $>500 \mathrm{~m}$ per dope), with the fiber passing through baths of aqueous solutions that aid HFIP removal and protein secondary structure formation $^{[10]}$. The synthetic silks generated by this machine from recombinant protein produced by transgenic goats and their comparison to natural dragline silk are the focus of the current study.

In a previous study, it has been noted that the process pathway of spinning the synthetic silk will have great influence on the final material properties ${ }^{[10]}$. Some of these properties of the natural and synthetic spider silks have been reported in literature (mechanical ${ }^{[11,20]}$ and thermal ${ }^{[21,22]}$ ), but the pyroelectric response of silk is lacking in the literature. To this end, the thermal diffusivity, thermal conductivity, and local pyroelectric response have been measured in the current study for different post-extrusion processes of synthetic spider silk. This is the first time the mechanical, thermal, and pyroelectric behavior of both natural and synthetic dragline silk have been investigated in the literature and compared.

This study focuses on the effect of processing conditions on the thermal and mechanical properties of synthetic silk, created from spidroin proteins from transgenic goat milk by means of a spinning process that makes use of a modified plastic extruder. These properties 


\section{WILEY-VCH}

are correlated to each other, to their pyroelectric response, and to the final diameter of the fiber. Literature values and diagrams of the crystalline alignment from XRD are also compared to thermal diffusivity and fiber diameter. These observations between the different material characteristics provide insight into processing aspects that improve the synthetic silk's properties and a potential mechanism is proposed to explain why synthetic silks have lower thermal and mechanical properties than natural silks.

\section{Experimental Description}

This section covers the formation and preparation of the silk samples as well as the material characterization setups.

\subsection{Nephila clavipes Silk Collection}

Silk was collected from adult Nephila clavipes spiders, which originated from the state of Florida, US. Silk was collected using the method described by Xu et al. ${ }^{[14]}$ Briefly, spiders were anesthetized by exposing them to $\mathrm{CO}_{2}$, allowing them to be restrained on the top of a Petri dish. With a dissecting microscope, the spinnerets for dragline silk were located and then, using tweezers, the silk was teased out of the major ampullate gland. The silk was attached to a rotating spool and approximately 100 meters of silk was gathered, at a silking rate of about $2 \mathrm{~m} / \mathrm{min}$. To prevent dehydration, the spiders were misted during the spooling process.

\subsection{Synthetic Silk Production}

Recombinant spider silk protein was produced using the method described by Tucker et. $\mathrm{al}^{[9]}$. Briefly, spider silk protein is produced by transgenic goats. Goats produce either the MaSp1 protein or MaSp2 protein when they lactate, and the proteins are found in their milk. Each protein is approximately $65 \mathrm{kDa}$ long and contains the non-repetitive C-terminal and the repetitive region of the protein. Milk was defatted and then combined with $0.1 \mathrm{M}$ L-arginineHCL (Spectrum Chemical MFG. Corp., Gardena, CA) in a 1:1 ratio. The $\mathrm{pH}$ was adjusted to 9, the milk was mixed for 30 minutes, and then loaded on a custom system of tangential flow filters. For 24 hours, the milk was filtered and the concentrated whey was collected. Ammonium sulfate was added to the whey at a concentration of $1.2 \mathrm{M}$ and then mixed for 24 hours to salt out the protein. The protein was then serially washed and centrifuged with distilled water until the electrical conductivity of the runoff was less than $20 \mu \mathrm{S}$. The protein was then lyophilized.

Spider silk fibers were produced using the recombinant spider silk protein according to the method described by Copeland et. $\mathrm{al}^{[10]}$. Briefly, a spin dope was prepared by placing MaSp1 and MaSp2 proteins in a 4mL vial with a Teflon lid (Waters Associates, Milford MA). The two proteins were mixed in the same ratio as has been observed in the native Nephila clavipes spider $^{[23]}$, i.e. a 4:1 ratio of MaSp1 and MaSp2. HFIP (Oakwood Chemical, West Columbia, SC) and 88+\% formic acid (Alfa Aesar, Ward Hill, MA) were added to the vial in a ratio of $4: 1(\mathrm{v} / \mathrm{v})$. The final concentration of the protein in solution was $25 \%(\mathrm{w} / \mathrm{v})$. The solution was mixed by slowly rotating for 48 hours until all the protein was dissolved and the spin dope was observed to be homogenous. Impurities were removed by centrifuging the vials at 4180 rcf for 24 hours and pouring the spin dope into a new vial. The spin dope was loaded into a $1 \mathrm{~mL}$ Hamilton syringe (Hamilton Company, Reno, NV) with $10 \mathrm{~cm}$ of PEEK tubing (internal diameter 0.005”) (SUPELCO, Bellefonte, PA) attached for tubing. The syringe was placed in a modified DACA Spinline (DACA Instruments, Santa Barbara, CA) and extruded at a rate of $0.7 \mathrm{~mm}$ per minute into a coagulation bath comprised of $100 \%$ IPA (Pharmo-Products Inc., Brookfield CT). As-spun (or extruded) fibers were collected at this stage of the process. 


\section{WILEY-VCH}

1 Single stretched fibers were collected after being run through two sets of Godets with an

2 alcohol/water stretch bath between them. Double stretched fibers went through the same set of 3 Godets as the first bath but were then stretched again in a distilled water bath. A schematic of 4 the processing equipment is also provided in Figure 2 and by Copeland et. $\mathrm{al}^{[10]}$.

$\frac{\overline{\Delta T}}{\overline{\Delta T_{s}}}=\frac{\Delta V}{\Delta V_{s}}=1-\frac{96}{\pi^{4}} \sum_{m=1}^{\infty} \frac{e^{-(2 m-1)^{2} \pi^{2} \alpha t / L^{2}}}{(2 m-1)^{4}}$

$k=\frac{I^{2} R_{0} R^{\prime} L}{3 \pi D^{2}\left(R_{s}-R_{0}\right)}$

Because of the non-uniform geometries of processed synthetic spider silks (seen in the SEM images of Figure 3), determination of their thermal conductivity was not possible. To that end, the best approach is to determine the diffusivity of multiple fibers that underwent the same production process, assume that they have the same radiation characteristics, and curve-fit the property values as a function of the square of the length to determine a "zero-length" property. This "zero-length" value is where radiation heat loss would not over-predict the property measurement ${ }^{[21]}$ and provides an accurate thermal diffusivity value.

\subsection{Pyroelectric Setup}

A scanning pyroelectric microscope (SPEM) measurement results in a pyroelectric response distribution map that can be directly correlated to the local spontaneous polarization distribution. SPEM is a proven technique ${ }^{[26]}$ for the determination of a polarization distribution map with both lateral and depth resolution by scanning the laser beam over the sample surface and modulating the laser output at different frequencies. This method has been used successfully for pyroelectric crystals with dimensions on the order of tens to hundreds of micrometers.

Briefly, SPEM involves a modulated laser beam that induces a local harmonic change in temperature. If the illuminated region is pyroelectric, it generates a small electric current, which can then be measured by two parallel plate electrodes. The pyroelectric current vector is proportional to the local electrical polarization vector, and by scanning the laser beam over 


\section{WILEY-VCH}

different regions of the sample, the polarization distribution map can be obtained. The power modulation of the laser output is typically on the order of 10 to $100 \mathrm{~mW}$ peak-to-peak power, with laser beam spot sizes ranging from 20 to 1 micrometers in diameter (depending on the optics and laser source used). The pyroelectric current that is generated is on the order of femtoamperes $\left(10^{-15} \mathrm{~A}\right)$. To this end, a series of noise-reducing techniques have been employed and are explained in more depth in $\operatorname{Ref}^{[26]}$.

The local macroscopic polarization is dependent on both the local symmetry and the dipole moment of the oriented molecules, which in this case is spidroin crystallites. Because of this, the SPEM image is also indicative of changes in macroscopic orientation in the same sample. The pyroelectric response can then be used to compare the degree of macroscopic orientation between different samples if they are composed of the same material, which is the case with the spider silks investigated in this study. Having the same ratio of MaSp1 and MaSp2 proteins $(80: 20)$ in all synthetic fibers, the differences in the silk are merely due to the process they went through to be produced.

\subsection{SEM Setup}

SEM images were taken with a FEI Quanta FEG 650 microscope, at energies of 15 and $30 \mathrm{kV}$, after being placed on carbon tape and coated with $10 \mathrm{~nm}$ of gold. The fibers imaged were taken from the same strand of silk as the fibers used for thermal, pyroelectric, and mechanical testing. Three images of each fiber were taken and the diameter was measured at two locations on the fiber in each image (Figure 3).

\subsection{XRD}

X-ray fiber diffraction was performed on the BioCars 14bm-C beamline on samples measured at the Advanced Photon Source located at Argonne National Laboratory, Argonne IL, USA. Fibers were mounted and placed at a distance of $300 \mathrm{~mm}$ from the detector. Stretched fibers were placed with the stretched axis normal to the beam line. For a single image, data collection times were 60 seconds and ten images were taken for each sample. Background images were taken right after each sample was completed with identical parameters. Images were then processed using Fit2D software. XRD results have been presented previously in literature $^{[10]}$, and the improvement on crystallinity and axial alignment of fibers observed by XRD during processing of the fibers agrees well with the results from the current study. In the following, XRD diffraction patterns will be presented with the thermal diffusivity and fiber diameter results.

\section{Results}

\subsection{Natural Dragline Silk from Nephila clavipes}

Natural spider silk has received significant consideration in the literature, because of its favorable materials properties. Of particular interest is the dragline silk from the Nephila clavipes, with high strength, elongation, and toughness for a polymer and low density material $^{[14]}$. These values are typically in the range of $0.7-1.4 \mathrm{GPa}{ }^{[20,27,28]}$ for ultimate tensile strength, 6-35 \% for elongation ${ }^{[20,29-31]}$, and 100-400 $\mathrm{kJ} \mathrm{kg}^{-1}$ for toughness or energy-tobreak $^{[27,31]}$.

In the literature, initial studies on the thermal properties of dragline silk reported an exceptionally high thermal conductivity, comparable to copper, which improved upon stretching ${ }^{[32]}$. Originally, these high values were attributed to the crystalline $\beta$-sheets that exist in the natural dragline silk (as shown by $\mathrm{XRD}^{[33]}$ ). However, further investigation of the 


\section{WILEY-VCH}

1 thermal diffusivity ${ }^{[21,34]}$ and thermal conductivity ${ }^{[21]}$ of the natural dragline silk demonstrated that the initial results were erroneous, due to neglecting the radiation heat transfer contribution, to insufficient vacuum to eliminate convection, and to length dependence of the thermal property measured. The results presented in the current study take these additional considerations into account, thus yielding the additional accuracy needed to compare the natural to the synthetic spider silk. The value found for the thermal conductivity of dragline silk is $1.2 \mathrm{~W} \mathrm{~m}^{-1} \mathrm{~K}^{-1}$ and for the thermal diffusivity $6 \times 10^{-7} \mathrm{~m}^{2} \mathrm{~s}^{-1}$, with $12 \%$ uncertainty ${ }^{[21]}$. These values are considerably more reasonable for a semi-crystalline, protein-based polymer, such as human hair ${ }^{[35]}$ or a different species of spider ${ }^{[34]}$. Additionally, molecular dynamics simulations have shown that the $\beta$-sheets (that were the original explanation for the high conductivity of the dragline silk) have a thermal conductivity of only $2-4 \mathrm{~W} \mathrm{~m}^{-1} \mathrm{~K}^{-1}$, depending on the number of strands in the sheet ${ }^{[36]}$.

The polarization distribution of natural spider silk, measured using SPEM, displays a pyroelectric response in the radial orientation (Figure 4). This observation implies a macroscopic orientation of the spidroin crystallites, probably caused by the extrusion via the spinneret. This correlates with XRD and NMR results looking at the structure of natural spider silk ${ }^{[37-39]}$. It is possible that the pyroelectric and (inferred via symmetry properties) piezoelectric properties potentially serve a physiological function, serving as temperature and/or pressure sensors (as hypothesized for the chitin wings of certain insects ${ }^{[40]}$ ).

\subsection{Extruded (As-Spun) Synthetic Spider Silk}

The as-spun synthetic spider silk provides a baseline to compare the effect of processing on synthetic spider silks, because it contains the necessary protein content, but does not have the same microstructure as other silks. This is demonstrated by the severe brittleness of the fibers as they break easily when handled. Furthermore, XRD results in the literature have shown that the as-spun fiber has no appreciable crystalline content ${ }^{[10,11]}$.

The thermal properties of the as-spun synthetic spider silk were measured by the improved TET technique. Because of their uniform surface geometry, the thermal conductivity and thermal diffusivity can both be determined. The magnitudes of these values are $0.25 \mathrm{~W} \mathrm{~m}^{-1} \mathrm{~K}^{-}$ ${ }^{1}$ for the thermal conductivity and $1.68 \times 10^{-7} \mathrm{~m}^{2} \mathrm{~s}^{-1}$ for the thermal diffusivity, similar to synthetic films measured in Ref. ${ }^{[41]}$. This is almost 6 times lower than the thermal transport values measured for natural spider silk. These significantly lower properties are also observed in the mechanical properties (32.5 MPa for ultimate tensile strength, $1 \%$ for extensibility, and $0.18 \mathrm{~kJ} \mathrm{~kg}^{-1}$ for toughness), and this decrease is attributed to the lack of crystallinity that exists based on XRD investigation of the silks ${ }^{[11]}$. The XRD diffraction pattern indicates weak alignment of the spidroin protein crystallites which is in line with the pyroelectric measurement (a direct indication of local macroscopic alignment) and the low thermal diffusivity and conductivity.

The pyroelectric SPEM response map of extruded synthetic silk (Figure 5, top) primarily displays an axial polarization component, whereas natural silk has a dominating radial component. Unlike the natural silk, the as-spun synthetic silk displays a coaxial polarization, seen primarily with the low frequency image that represents structure deeper in the fiber (Figure 5, top right). The axial alignment arises from the axial extrusion process, forcing the synthetic spidroin crystallites in a macroscopic axial orientation. The coaxial behavior could be due to a number of effects, the most likely being the existence of a thermal gradient in the thick synthetic silk fiber during the sol-gel transition. A high frequency pyroelectric scan which represents surface behavior reveals several domains in the axial orientation (Figure 5, 


\section{WILEY-VCH}

top left), corresponding to the rough texture seen in the SEM images. This could well be the result of a polar self-assembly process during the extrusion ${ }^{[42]}$.

Further stretching and processing of the extruded silk fiber could result in greater macroscopic alignment of the crystallites, yielding thermal and mechanical properties closer to the ones of natural silk.

\subsection{Processed Synthetic Silk}

The thermal diffusivity values of several synthetic spider silks with different processes have been measured. The non-uniformities of the synthetic spider silks' geometry would add significant errors to the thermal conductivity measurement ${ }^{[21]}$ because of the need for an accurate volumetric heating in the model, so the thermal conductivity is not reported. However, the sensitivity of the thermal diffusivity (proportional to the thermal conductivity by the expression $\alpha=k / \rho c_{p}$ ) to processing variations should be sufficient as a metric because the diffusivity measurement is not affected by sample volume. To that end, eight different processed silks have been measured, based on different processing variations of the spinning apparatus shown in Figure 2. These variations fall into the following categories: (1) \% volume of solution contents in solution bath $\mathrm{B}-\mathrm{IPA} / \mathrm{H}_{2} \mathrm{O} / \mathrm{MeOH}$, (2) use of pure water bath $\mathrm{D}$ for dipping or stretching, and (3) extent of stretching in the liquid baths that the fiber passes through $-2 \mathrm{x}, 3 \mathrm{x}$. Their properties and pyroelectric response are best compared to each other and to the natural spider silk they are meant to mimic in the next section.

\subsection{Comparison between Natural and (Processed) Synthetic Silk}

When comparing extruded synthetic silk to further processed silk, a favorable increase in the investigated material properties is observed. Further processing of the extruded silk results in increased thermal diffusivity, predicted thermal conductivity, pyroelectric response, and macroscopic crystalline $\beta$-sheet orientation.

Figure 6 and Figure 7 show the relationship between the thermal diffusivity of each silk compared to its mechanical properties and pyroelectric response. There is a noticeable correlation: an improvement in one property is mirrored in an improvement of the other properties. This is indicative of improvements in the organization of the silk's microstructure, as crystalline $\beta$-sheets are formed upon passing of the fiber through the solution baths (shown by $\mathrm{XRD}^{[10]}$ and Figure 8) and increased axial alignment of the dipoles due to the stretching (shown by SPEM, Figure 5, bottom).

It is interesting to note that the increase in material properties has an inverse correlation to the silk's diameter, i.e. a decrease in diameter corresponds to an increase in thermal diffusivity or pyroelectric response (Figure 8). It is likely that the repeated stretching and solution-dipping (which results in a smaller diameter) also reduces the radial gradient in material properties, as evidenced by the pyroelectric data. This produces a fiber that is more uniform in both the axial and radial directions and better resembles the crystallites' orientation in native silk. As stretching causes elongation in one direction, conservation of mass and density dictates that the other dimensions should shrink. In addition, this stretching causes flow alignment of the crystallites, increasing macroscopic orientation. However, there is a limit to the amount of stretching and diameter reduction that can occur without failure of the fiber. One potential option for smaller diameter fiber would be to use a use a smaller diameter extrusion outlet. However, with smaller diameter extrusion the chance for breaks in the fiber during production are higher for even the smallest non-homogeneity in the spin dope. These potential 


\section{WILEY-VCH}

1 inhomogeneities could be overcome with an increase of the protein size to more closely

2 mimic natural spider silk.

3

4

\section{Conclusions and Outlook}

The measurement of material strength, thermal diffusivity and local pyroelectric response for synthetic silk created by varying processes clearly indicates a positive correlation between these material properties and a decrease in the fiber's diameter.

Further processing and stretching of the extruded silk reduces its diameter, introducing an axial flow alignment of the spidroin crystallites. When the $\beta$-sheet spidroin crystallites enjoy a high degree of macroscopic orientation, they allow for a better transmission of heat conduction and increased tensile strength. The high degree of orientation is also responsible for a high spontaneous polarization and associated pyroelectric response to small thermal perturbations.

These findings (particularly the polarization findings by SPEM and potential radial gradient during fiber processing) imply that for the large scale production of synthetic spider silk that more closely mimics the behavior of the natural dragline silk, care must be taken to reduce the radial gradient of the fiber during processing. This work has demonstrated that this can be accomplished by stretching, but other mechanisms to decrease the fiber size could potentially improve the fibers' properties. Furthermore, control of the diameter could also provide the ability to tune the properties of the fiber for future applications.

\section{Acknowledgements}

The authors would like to acknowledge the support from KU Leuven research project OT/11/064 (Investigation of exotic thermal and elastic behavior of soft matter and functional thin layers by means of advanced experimental techniques), USTAR, the National Science Foundation, and the Department of Energy.

We acknowledge the support from the Microscopy Core Facility at Utah State University for the SEM work.

T. Munro, C. Copeland, and T. Putzeys contributed equally to this work.

Received: ((will be filled in by the editorial staff))

Revised: ((will be filled in by the editorial staff))

Published online: ((will be filled in by the editorial staff)) 


\section{WILEY-VCH}

\section{References}

[1] I. Agnarsson, M. Kuntner, T. A. Blackledge, PloS One 2010, 5, e11234.

[2] R. V. Lewis, Chem. Rev. 2006, 106, 3762.

[3] J. M. Gosline, M. E. DeMont, M. W. Denny, Endeavour 1986, 10, 37.

[4] J. M. Gosline, P. A. Guerette, C. S. Ortlepp, K. N. Savage, J. Exp. Biol. 1999, 202, 3295.

[5] C. Boutry, M. Řezáč, T. A. Blackledge, PloS One 2011, 6, e22467.

[6] J. G. Hardy, L. M. Römer, T. R. Scheibel, Polymer 2008, 49, 4309.

[7] X.-X. Xia, Z.-G. Qian, C. S. Ki, Y. H. Park, D. L. Kaplan, S. Y. Lee, Proc. Natl. Acad. Sci. 2010, 107, 14059.

[8] K. Costas N, T. Jeffrey D, K. Anthoula, Production of Biofilaments in Transgenic Animals, US Patent 7,157,615 2001.

[9] C. L. Tucker, J. A. Jones, H. N. Bringhurst, C. G. Copeland, J. B. Addison, W. S. Weber, Q. Mou, J. L. Yarger, R. V. Lewis, Biomacromolecules 2014, 15, 3158.

[10] C. G. Copeland, B. E. Bell, C. D. Christensen, R. V. Lewis, ACS Biomater. Sci. Eng. 2015.

[11] F. Teulé, B. Addison, A. R. Cooper, J. Ayon, R. W. Henning, C. J. Benmore, G. P. Holland, J. L. Yarger, R. V. Lewis, Biopolymers 2012, 97, 418.

[12] B. An, M. B. Hinman, G. P. Holland, J. L. Yarger, R. V. Lewis, Biomacromolecules 2011, 12, 2375.

[13] A. E. Albertson, F. Teulé, W. Weber, J. L. Yarger, R. V. Lewis, J. Mech. Behav. Biomed. Mater. 2014, 29, 225.

[14] M. Xu, R. V. Lewis, Proc. Natl. Acad. Sci. 1990, 87, 7120.

[15] M. B. Hinman, R. V. Lewis, J. Biol. Chem. 1992, 267, 19320.

[16] J. A. Jones, T. I. Harris, C. L. Tucker, K. R. Berg, S. Y. Christy, B. A. Day, D. A. Gaztambide, N. J. Needham, A. L. Ruben, P. F. Oliveira, others, Biomacromolecules 2015, 16, 1418.

[17] Q. Peng, Y. Zhang, L. Lu, H. Shao, K. Qin, X. Hu, X. Xia, Sci. Rep. 2016, 6, 36473. [18] A. Heidebrecht, L. Eisoldt, J. Diehl, A. Schmidt, M. Geffers, G. Lang, T. Scheibel, Adv. Mater. 2015, 27, 2189.

[19] S. Rammensee, U. Slotta, T. Scheibel, A. Bausch, Proc. Natl. Acad. Sci. 2008, 105, 6590.

[20] P. M. Cunniff, S. A. Fossey, M. A. Auerbach, J. W. Song, D. L. Kaplan, W. W. Adams, R. K. Eby, D. Mahoney, D. L. Vezie, Polym. Adv. Technol. 1994, 5, 401.

[21] C. Xing, T. Munro, B. White, H. Ban, C. Copeland, R. Lewis, Polymer 2014, 55, 4226. [22] W. Huang, S. Krishnaji, X. Hu, D. Kaplan, P. Cebe, Macromolecules 2011, 44, 5299. [23] A. E. Brooks, H. B. Steinkraus, S. R. Nelson, R. V. Lewis, Biomacromolecules 2005, 6, 3095.

[24] C. Xing, T. Munro, C. Jensen, H. Ban, Meas. Sci. Technol. 2013, 24, 105603.

[25] C. Xing, T. Munro, C. Jensen, B. White, H. Ban, C. G. Copeland, R. V. Lewis, Meas. Sci. Technol. 2014, 25, 115604.

[26] T. Putzeys, M. Wübbenhorst, Dielectr. Electr. Insul. IEEE Trans. On 2012, 19, 1186.

[27] S. Mukhopadhyay, J. Sakthivel, J. Ind. Text. 2005, 35, 91.

[28] R. S. Rengasamy, M. Jassal, C. Rameshkumar, AUTEX Res J 2005, 5, 30.

[29] D. O’Sullivan, Chem. Eng. News 1988, 66, 24.

[30] Y. Yang, X. Chen, Z. Shao, P. Zhou, D. Porter, D. P. Knight, F. Vollrath, Adv. Mater. 2005, 17, 84.

[31] J. M. Gosline, M. E. DeMont, M. W. Denny, Endeavour 1986, 10, 37.

[32] X. Huang, G. Liu, X. Wang, Adv. Mater. 2012, 24, 1482. 


\section{WILEY-VCH}

1 [33] A. Bram, C. Bränden, C. Craig, I. Snigireva, C. Riekel, J. Appl. Crystallogr. 1997, 30, 390.

[34] R. Fuente, A. Mendioroz, A. Salazar, Mater. Lett. 2014, 114, 1.

[35] A. Salazar, A. Mendioroz, R. Celorrio, others, J. Appl. Phys. 2010, 107, 043508.

[36] L. Zhang, T. Chen, H. Ban, L. Liu, Nanoscale 2014, 6, 7786.

[37] S. Sampath, T. Isdebski, J. E. Jenkins, J. V. Ayon, R. W. Henning, J. P. R. O. Orgel, O. Antipoa, J. L. Yarger, Soft Matter 2012, 8, 6713.

[38] G. P. Holland, M. S. Creager, J. E. Jenkins, R. V. Lewis, J. L. Yarger, J. Am. Chem. Soc. 2008, 130, 9871.

10 [39] M. S. Creager, J. E. Jenkins, L. A. Thagard-Yeaman, A. E. Brooks, J. A. Jones, R. V. 11 Lewis, G. P. Holland, J. L. Yarger, Biomacromolecules 2010, 11, 2039.

12 [40] T. Putzeys, M. Wubbenhorst, Dielectr. Electr. Insul. IEEE Trans. On 2015, 22, 1394.

13 [41] S. Xu, Z. Xu, J. Starrett, C. Hayashi, X. Wang, Polymer 2014, 55, 1845.

14 [42] J. Hulliger, Chem. - Eur. J. 2002, 8, 4578.

15 [43] S. Asha, Y. Sangappa, S. Ganesh, J. Spectrosc. 2015, 2015. 


\section{WILEY-VCH}

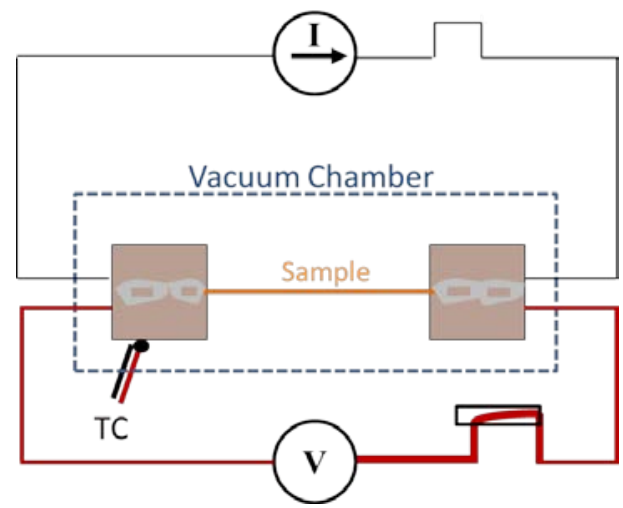

Figure 1. Schematic of improved TET method showing step current input and voltage response of sample.

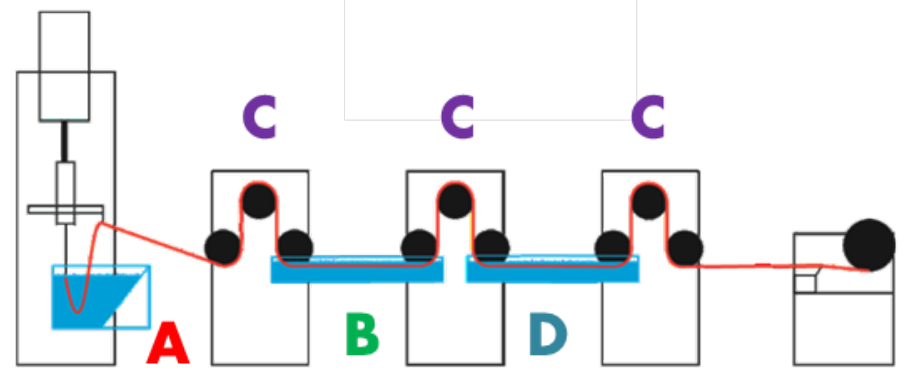

Figure 2. Synthetic spider silk spinning apparatus, (A) is coagulation bath, (B) is initial solution bath (either IPA/ $\mathrm{H}_{2} \mathrm{O}$ or $\mathrm{MeOH} / \mathrm{H}_{2} \mathrm{O}$, with details provided in the text), (C) are godets for stretch rate control, and (D) is pure DI water bath.
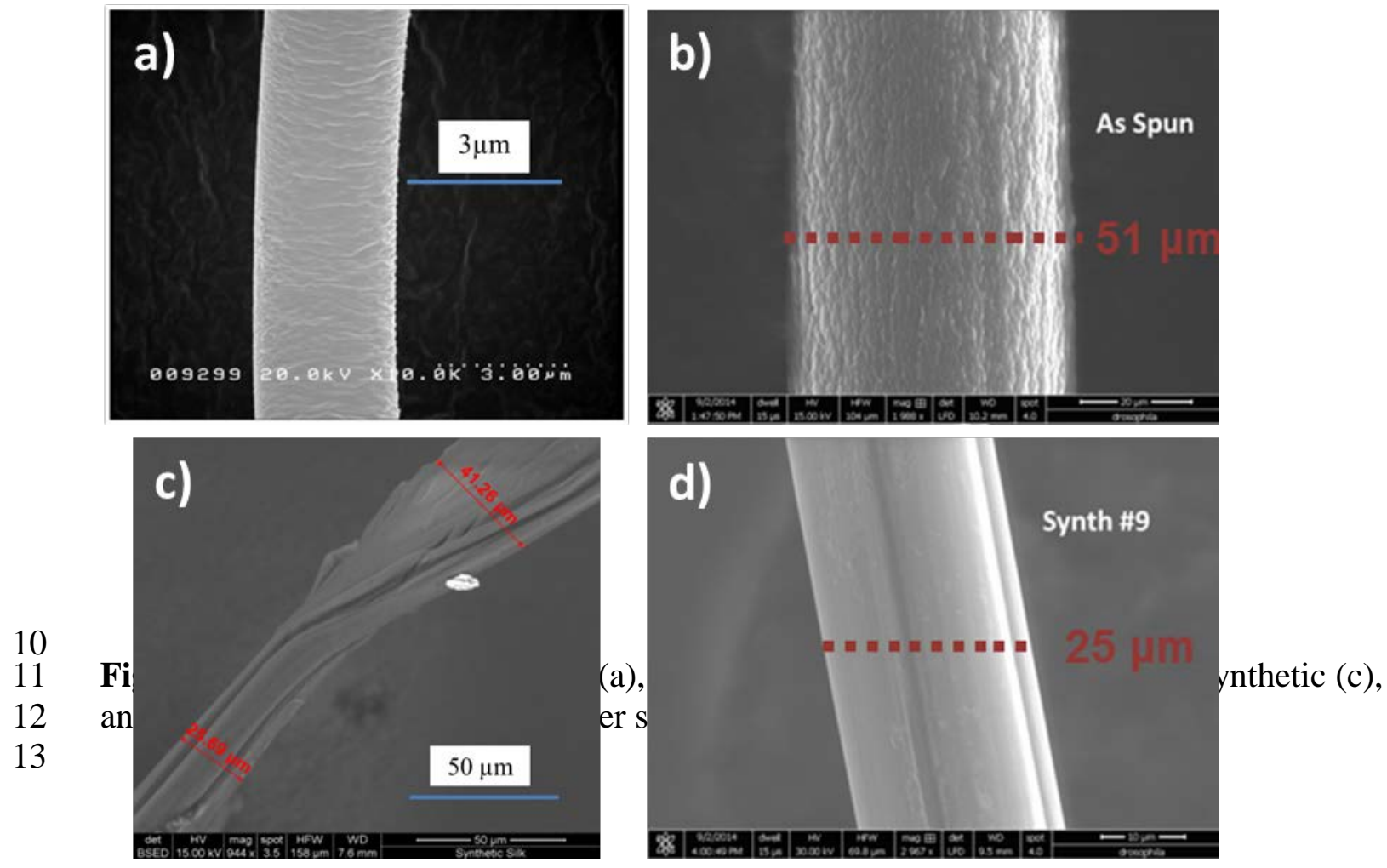


\section{WILEY-VCH}

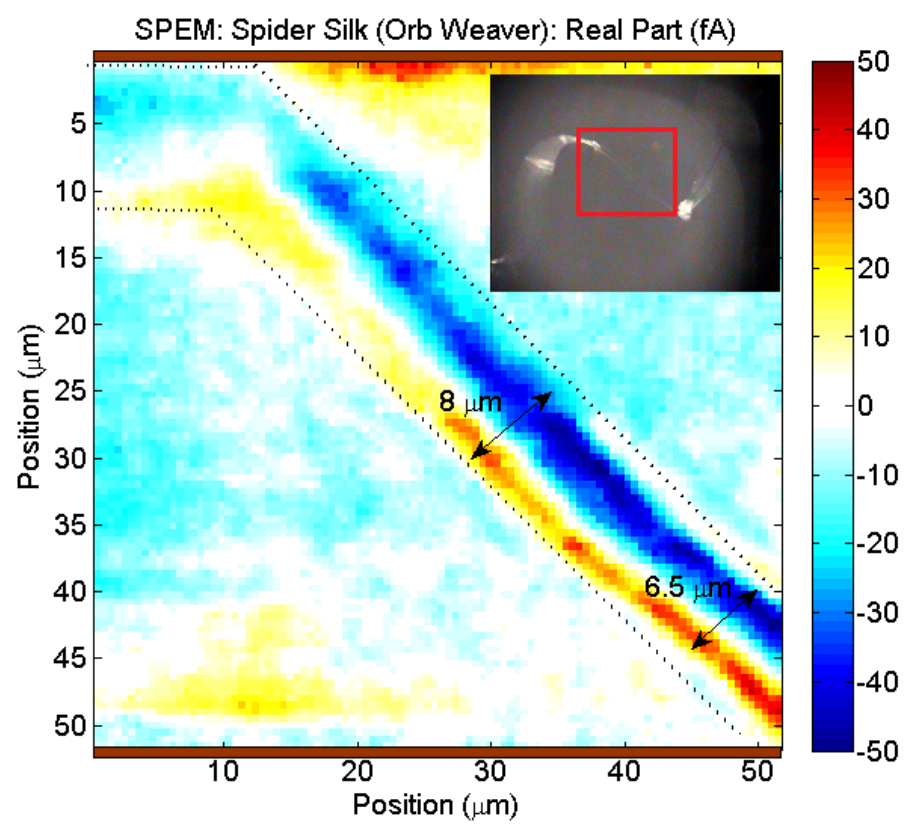

Figure 4. SPEM image of natural dragline silk.
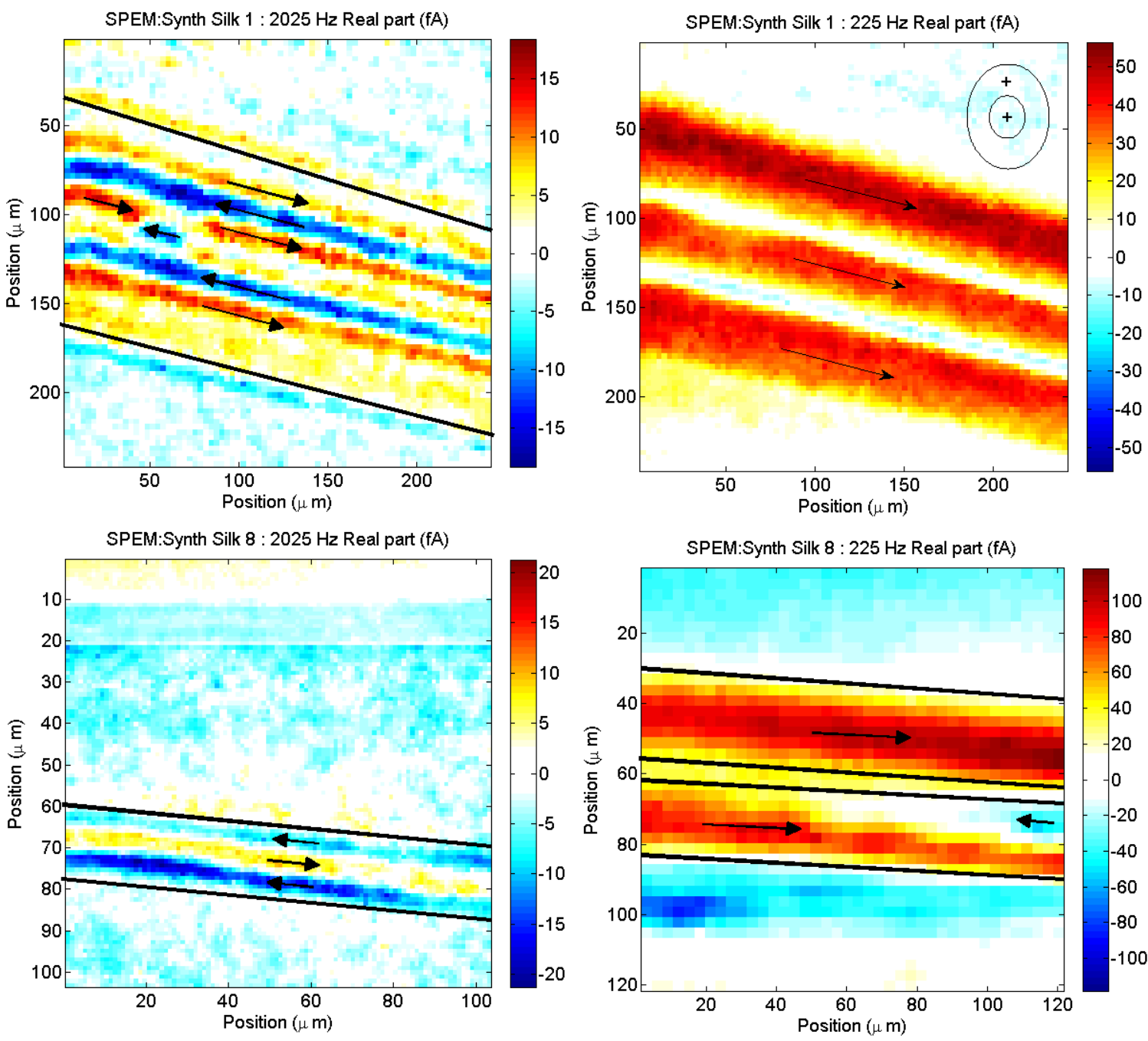

Figure 5. SPEM dipole orientations of extruded synthetic silk fiber (top) compared to processed synthetic silk fiber (bottom). Images on the left are at high frequencies and are representative of behavior near the surface, while images on the right are at low frequencies 8 and are representative of behavior deeper inside the fiber. 

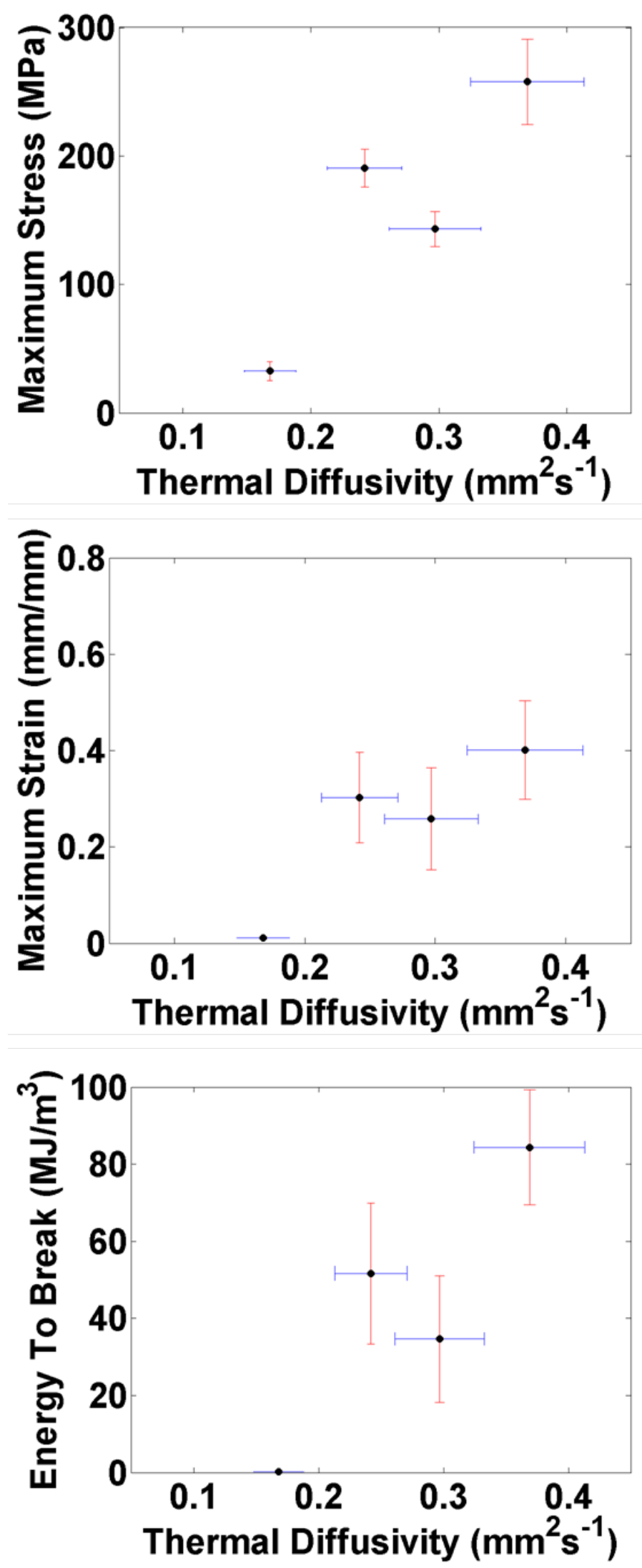

3 Figure 6. Correlation between the thermal diffusivity and different mechanical properties, 4 measured in different processing conditions: ultimate tensile strength (top), maximum 5 extensibility (middle), and energy to break (bottom), showing the trend that processing of the 6 silk (via stretching and passing through water baths), improves both properties. 
WILEY-VCH

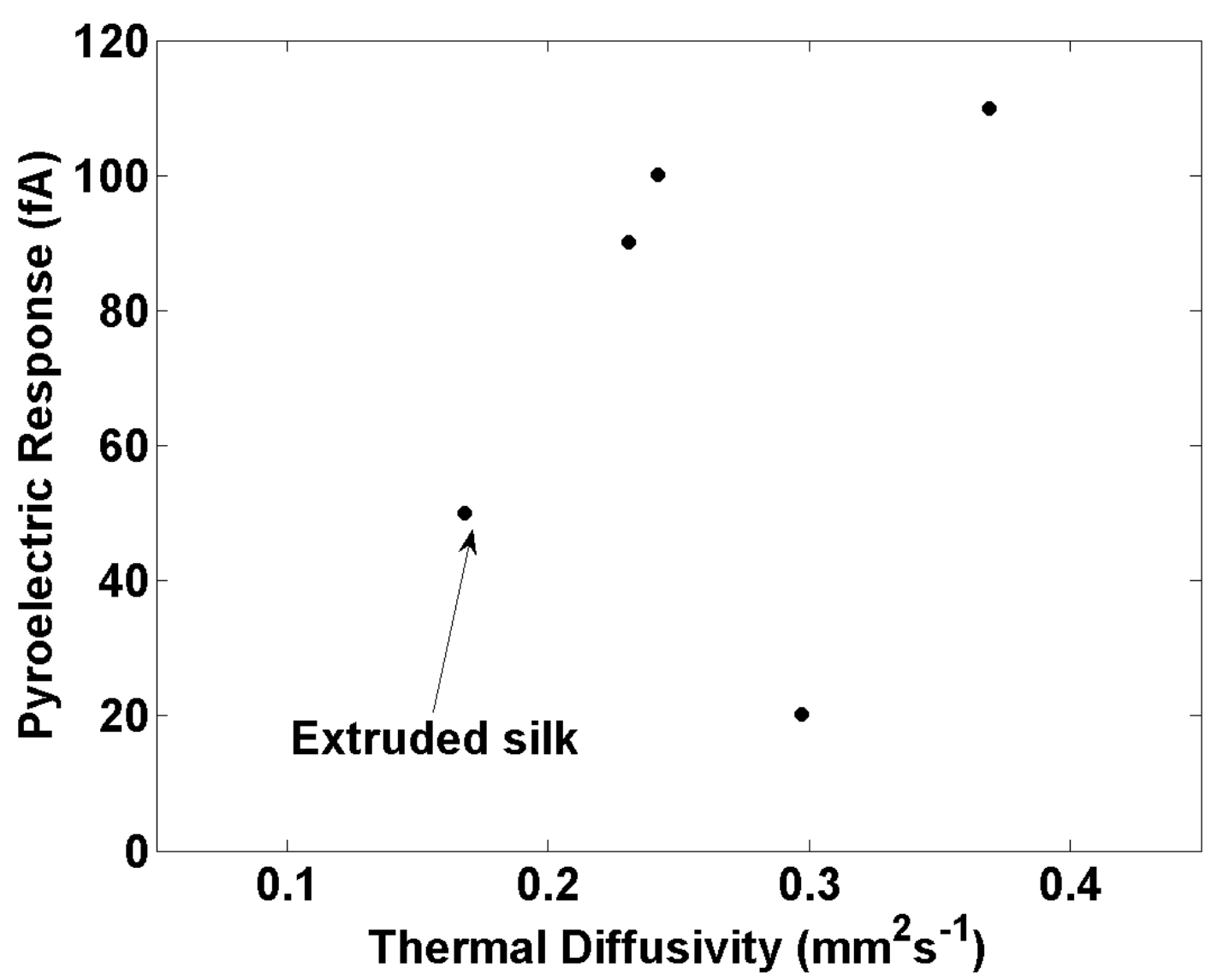

Figure 7. Pyroelectric current of spider silk compared to thermal diffusivity (based on laser intensity modulated between $10-90 \mathrm{~mW}$, and a sample reflectivity between $10-30 \%{ }^{[43]}$ ). The presence and macroscopic alignment of crystalline sheets in the synthetic silk contribute to a greater pyroelectric response, which is also mirrored in the thermal diffusivity. 


\section{WILEY-VCH}

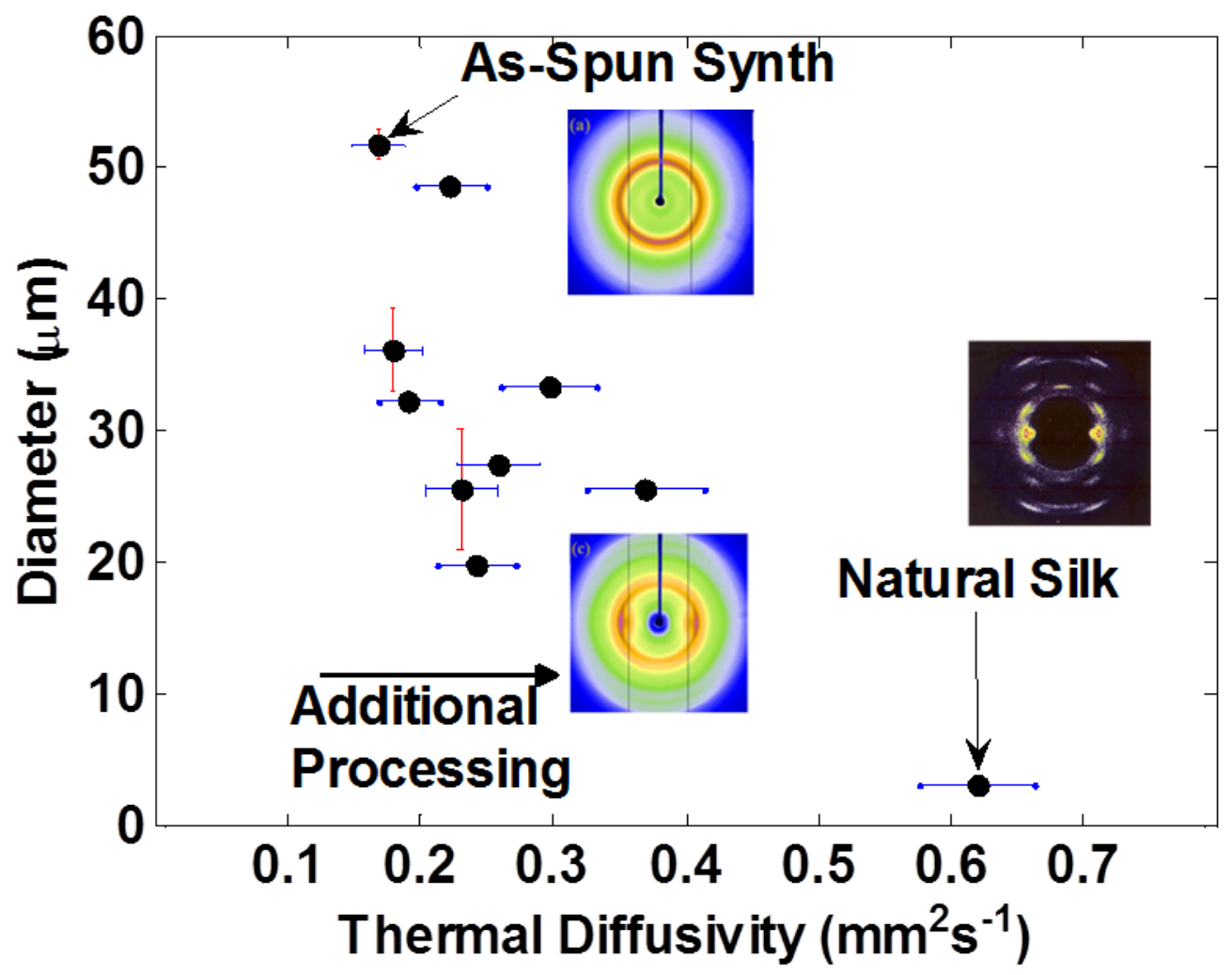

Figure 8. Thermal diffusivity of the natural dragline and synthetic spider silks, showing the trend to smaller diameters and improved thermal property as the fiber is put through additional processing treatments (multiple stretchings, multiple liquid baths). XRD diffraction patterns reprinted with permission from Refs. ${ }^{[\mathbf{1 0}, 33]}$, copyright 2015 and 1997 respectively. 


\section{WILEY-VCH}

1 A correlation between the thermal properties, mechanical properties, pyroelectric

2 response, and diameter of natural and synthetic spider silk is observed for the first time.

3 The results show that the processes during the synthetic fiber production induce crystal

4 formation and axial alignment, but the fibers still have different structural organization than

5 natural silks. (XRD diffraction patterns reprinted with permission from Refs. ${ }^{[10,33]}$, copyright

62015 and 1997 respectively).

7

Keyword: spider silk, strength, toughness, thermophysical, pyroeletric

T. Munro*, T. Putzeys, C. Copeland, C. Xing, R. Lewis, H. Ban, C. Glorieux, M. Wubbenhorst

Title: Investigation of synthetic spider silk crystallinity and alignment via electrothermal,

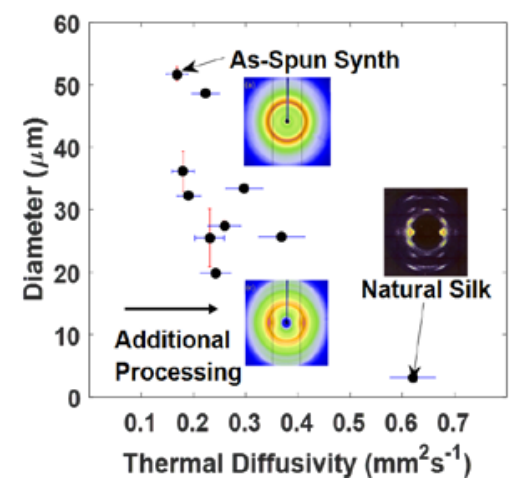

